\title{
Sex Differences in Hepatic Gluconeogenic Capacity After Chronic Alcohol Consumption
}

\author{
Ken D. Sumida, PhD; Janeen M. Hill, PhD; and Aleksey V. Matveyenko, PhD
}

\begin{abstract}
Alcohol-induced hypoglycemia has traditionally been attributed to the amount of ethanol consumed rather than any inherent decline in glucose output capacity by the gluconeogenic organs and/or an increase in skeletal muscle glucose uptake. Further, while the potential for sex differences that might impact glucose homeostasis following chronic alcohol consumption has been recognized, direct evidence has been noticeably absent. This paper will provide a brief review of past and present reports of the potential for sex differences in glucose homeostasis following chronic ethanol consumption. This paper will also provide direct evidence from our laboratory demonstrating sex differences from chronic alcohol consumption resulting in a decrement in glucose appearance and more importantly, a specific decline in hepatic gluconeogenic (HGN) capacity in the absence and presence of ethanol. All our studies involved 8 weeks of chronic alcohol consumption in male and female Wistar rats, as well as a 24 to 48 hour fast to deplete hepatic glycogen stores. Under the conditions of chronic alcohol consumption and an acute dose of ethanol, we provide in vivo evidence of an early decline in whole body glucose appearance in females fed an ethanol diet compared to controls. While the decline was also observed in males fed the alcohol diet, it occurred much later compared to ethanol fed females. The site for the decline in whole body glucose production (i.e., either the kidneys or the liver) was beyond the scope of our prior in vivo study. In a follow-up study using the in situ perfused liver preparation, we provide additional evidence for a specific reduction in HGN capacity from lactate in ethanol fed females compared to ethanol fed males in the absence of alcohol in the perfusion medium. Finally, employing the isolated hepatocyte technique, we report decrements in HGN from lactate in ethanol fed females compared to ethanol fed males in the presence of ethanol in the incubation medium. The mechanism for the specific decline in HGN within the liver of ethanol fed females remains to be determined. To the extent that our observations in animals can be extrapolated to humans, we conclude that alcoholic women are more susceptible to ethanol-induced hypoglycemia compared to alcoholic men.
\end{abstract}

Keywords: Alcohol-induced hypoglycemia; Glucose homeostasis; Males vs. females

\section{mpact of Acute and Chronic Alcohol} Consumption on Glucose Homeostasis

Blood glucose homeostasis is essential to ensure an adequate concentration difference for glucose extraction and use by the central nervous system. The maintenance of blood glucose levels is important given its role as the primary nutrient for energy production in the brain. A fall in blood glucose to below $55 \mathrm{mg} / \mathrm{dl}$ (i.e., approximately $3 \mu \mathrm{moles} / \mathrm{ml}$ ) significantly impairs both cognitive and motor function due to neuroglycopenia. ${ }^{1}$ Further declines in blood glucose to

Reprint Requests: Ken D. Sumida, PhD, Chapman University, Department of Biological Sciences, One University Drive, Orange, CA 92866, Tel: 7|4-997-6995, Fax: 7|4-532-6048, E-mail: sumida@chapman.edu below $30 \mathrm{mg} / \mathrm{dl}$ (i.e., $<2 \mu \mathrm{moles} / \mathrm{ml}$ ) may result in coma, seizures, neurologic dysfunction, and possibly, death. ${ }^{1}$

The primary organs responsible for blood glucose homeostasis are the kidneys and the liver through glycogenolysis and gluconeogenesis. The initial response to a decline in blood glucose concentration is the release of glucose from these organs via glycogenolysis. However, as the glycogen stores in these organs diminish, the kidneys and liver will rely more heavily on gluconeogenesis to prevent the onset of hypoglycemia. In a fasted state where both renal and hepatic glycogen stores are limited, the gluconeogenic

Grant Support: Supported by Grant AA II259-02 from the National Institute on Alcohol Abuse and Alcoholism. 
capacity within these organs are elevated, making it the primary mode by which blood glucose concentrations are maintained. Given the importance of both the kidneys and liver in blood glucose homeostasis, a decline in glucose output capacity within either of these organs could result in hypoglycemia and its associated effects.

Freinkel et $\mathrm{a}^{2}$ were among the first investigators to report that acute doses of ethanol (hereafter, and perhaps inappropriately, referred to as "alcohol") can result in a significant decline in blood glucose levels. Further studies by Krebs and coworkers ${ }^{3,4}$ determined that the oxidation of ethanol within the liver through the nicotinamide adenine dinucleotide $\left(\mathrm{NAD}^{+}\right)$-dependent alcohol dehydrogenase $(\mathrm{ADH})$ pathway generates an elevation of cytosolic NADH, thereby increasing the NADH/NAD ${ }^{+}$ratio. The shift in the redox state supports a possible mechanism for the inhibition of hepatic gluconeogenesis (HGN) and corroborates the occurrence of alcohol-induced hypoglycemia, especially in malnourished individuals where renal and hepatic glycogen stores are compromised. While not a consistent observation, reports in fasted humans $\mathrm{s}^{5-7}$ and fasted rats ${ }^{8-10}$ have demonstrated a significant decline in blood glucose concentration after a substantial ingestion of ethanol.

While much is known about the inhibitory effects of acute alcohol consumption on HGN, the impact of chronic alcohol consumption remains to be elucidated. Knowledge of the impact of chronic ethanol ingestion on HGN is of considerable importance given that some alcoholics tend to reduce their food intake and/or consume diets low in carbohydrates. ${ }^{11,12}$ Under these circumstances of fasting or inadequate nutritional intake, renal and hepatic glycogen stores would be diminished. Thus for the alcoholic, if there was a concomitant decrement in gluconeogenic capacity due to chronic alcohol consumption, this could result in a greater susceptibility for alcohol-induced hypoglycemia.

Other contributing factors for the hypoglycemic effects associated with alcohol consumption involve its impact upon insulin secretion and lipolysis. An acute over-consumption of ethanol has been observed to elevate glucose-induced insulin secretion. ${ }^{13}$ In addition, more recent studies report that alcohol results in the reduction of circulating plasma free fatty acid levels. ${ }^{14}$ The deleterious effect of low free fatty acid levels is two-fold: first, lowering the availability of fats elevates the reliance on glucose; and second, fatty acids have been shown to stimulate HGN. ${ }^{15}$ Thus, lower plasma fatty acid levels will attenuate the permissive action on glucose production capacity. Collectively, the elevated glucoseinduced insulin secretion, the lower levels of free fatty acids, and the inhibitory effects observed with acute ethanol upon HGN would increase glucose uptake and simultaneously lower glucose production.

The impact of chronic alcohol consumption on insulin sensitivity and circulating triglycerides is more complex.
Recent studies report that light to moderate alcohol consumption can lower the prevalence of metabolic syndrome. ${ }^{16,17}$ Briefly, features of metabolic syndrome include abdominal obesity, low serum high-density lipoprotein (HDL) cholesterol, hypertriglyceridemia, hyperglycemia and elevated blood pressure, partially due to insulin resistance but culminating in an elevated risk of cardiovascular disease and diabetes. ${ }^{16}$ In this regard, mild to moderate chronic alcohol consumption has been observed to elevate serum HDL cholesterol, lower circulating triglycerides, and while not a consistent observation, improve insulin sensitivity. ${ }^{16,17}$ However, in heavy drinkers, chronic alcohol consumption is associated with an elevated prevalence of metabolic syndrome. ${ }^{18}$ Thus, there appears to be multiple factors that dictate the subsequent impact of alcohol ingestion that are mediated by the specific effect of ethanol upon various organs, the amount of acute alcohol consumed, acute versus chronic ethanol ingestion, and the type of chronic alcohol consumption (i.e., light/moderate vs. heavy). Other related factors include age, ethnicity and fasting versus postprandial state, which are beyond the scope of this review.

\section{Alcohol Consumption and Sex Differences in Glucose Homeostasis}

Our understanding of the effects of alcohol consumption on glucose homeostasis is further complicated by differences in sex. For example, there are differences in the liver's response to both an acute and chronic consumption of ethanol in men versus women. Further, reports in humans suggest that there are sex differences in the location and quantity of hepatic $\mathrm{ADH}^{19}$ and in the first-pass metabolism of ethanol, the latter of which can give rise to higher blood alcohol levels in women compared to men despite an equivalent consumption of ethanol.20 Animal experiments of chronic alcohol consumption have demonstrated that females tend to accumulate more fatty acid within the liver than males. ${ }^{21}$ This observation in animals corroborates prospective studies demonstrating the greater likelihood of liver disease in women after consuming less ethanol and within a shorter time period compared to men. ${ }^{22}$

Despite the increased recognition of sex differences that can occur after chronic alcohol consumption, few investigations have examined the interaction of chronic alcohol consumption and gender in affecting HGN capacity and blood glucose homeostasis. The purpose of this paper is to provide a brief overview of past and present research that has examined this interaction. We will emphasize research findings from our laboratory using an animal model. Specifically, we will provide evidence supporting the hypothesis that chronic alcohol consumption leads to sex differences in HGN capacity that is more detrimental to female rather than male rats. An extrapolation of the work done in our laboratory suggests that the incidence of ethanolinduced hypoglycemia would conceivably be greater for alcoholic women compared to men. 
Several reasons are likely explanations for the lack of information about the impact of chronic alcohol consumption and gender on glucose homeostasis in humans. First, the use of clinical tests combined with a properly conducted interview is necessary to distinguish between the effects of acute versus chronic alcohol consumption on blood glucose concentration. The time involved to conduct an interview, and the intoxication level of the patient may conspire to reduce both the number and accuracy of incidence reports from hospitals documenting the occurrence of ethanol-induced hypoglycemia in male versus female alcoholics. Second, alcoholics may not be forthcoming because they may never seek medical assistance. Third, the prevailing hypothesis suggests that alcohol-induced hypoglycemia is dependent upon the amount of ethanol ingested within a short time rather than any specific impact from chronic alcohol consumption due to sex differences. As such, the potential for sex differences in glucose homeostasis after chronic alcohol consumption has been overlooked.

In contrast, a topic that has been extensively investigated as it pertains to sex differences and ethanol consumption is $\mathrm{ADH}$, the primary enzyme responsible for ethanol metabolism. The activity of this enzyme can impact the blood alcohol level following ethanol consumption, and as previously discussed, alcohol can attenuate hepatic glucose production and impact glucose homeostasis. In this regard, males tend to have higher gastric ADH activity compared to females, ${ }^{20}$ whereas females tend to have higher hepatic ADH activity compared to males. ${ }^{23}$ Of interest, women demonstrate higher blood alcohol levels compared to men, even when the alcohol ingestion per body weight is equivalent. ${ }^{24}$ In an elegant review by Lieber, ${ }^{25}$ the attributes of gastric ADH were compared to hepatic $\mathrm{ADH}$, where one of the gastric $\mathrm{ADH}$ isoenzymes not found in the liver has a significantly higher $\mathrm{V}_{\max }$, which has been offered as a mechanism to prevent the entry of alcohol into the body. The fact that men have higher gastric $\mathrm{ADH}$ activities results in a greater first-pass metabolism of alcohol compared to women and substantiates the higher blood alcohol levels observed in females. Further, the distribution space for alcohol is smaller in women compared to men. ${ }^{26}$ This also contributes to the higher blood alcohol levels observed in females. Following chronic alcohol consumption, however, the sex differences are even greater and more detrimental for women. For alcoholic men, the blood alcohol level achieved was significantly greater than for nonalcoholic men given the same dose of ethanol. ${ }^{26}$ For alcoholic women, the differences in blood alcohol levels were even greater compared to nonalcoholic women. ${ }^{26}$ In fact, alcoholic women appear to lose the gastric protective mechanism normally provided by the first-pass metabolism of alcohol and are dependent upon the liver for ethanol detoxification, the organ also responsible for glucose output. Finally, the difference in gastric ADH between nonalcoholic men and women appears to disappear after 50 years of age. ${ }^{27}$ The mechanism for the disappearance is unknown, but we would be remiss if we did not acknowledge that the age of an individual may be an additional factor to consider. In this regard, we also recognize that ethnicity is yet another factor that warrants consideration. As previously mentioned, both of these factors go beyond the scope of this review. In summary, following the ingestion of ethanol, the circulating blood alcohol level is greater in women compared to men and is exacerbated in alcoholic women vs. alcoholic men. Taken together, there appears to be a potential for sex differences that would impact the liver after chronic alcohol consumption, resulting in a detrimental effect upon glucose homeostasis.

\section{Evidence for Sex Differences in Liver Glucose Output}

The larger size of the liver compared to the kidneys portends its role as the primary organ responsible for glucose homeostasis. As such, most of the previous studies focus on the liver. A seemingly paradoxical observation in patients with alcohol-induced liver cirrhosis is an elevation in HGN capacity. ${ }^{28,29}$ Further, heavy ethanol ingestion for a prolonged period of time may also contribute to abdominal obesity and an increased risk for type 2 diabetes. ${ }^{30}$ Therefore, we have two contrasting effects related to alcohol consumption. Acute ethanol consumption can result in a decline in HGN, whereas liver cirrhosis due to alcohol abuse can culminate in an elevation in hepatic glucose production. In this regard, understanding the intermediary effects of chronic alcohol consumption and the impact of gender in the absence of cirrhosis and/or type 2 diabetes is essential. A decline in HGN capacity from three carbon precursors as a result of chronic ethanol consumption could elevate the risk of alcoholinduced hypoglycemia, while an increase in HGN capacity might lead to an earlier onset for glucose intolerance.

Winston and Reitz ${ }^{31}$ were among the first investigators to examine the liver as a possible factor that might impact glucose homeostasis in male versus female rats following chronic ethanol consumption. Using Long-Evans rats fed an alcohol diet for over 5 weeks, they reported more than a $60 \%$ decline in liver glycogen levels from both male and female animals chronically fed the ethanol diet compared to controls. ${ }^{31}$ While there was no significant difference in the reduced hepatic glycogen levels for alcohol fed males compared to females, the plasma glucose concentrations at the time of sacrifice were significantly lower for ethanol fed males compared to male controls. ${ }^{31}$ In contrast, there was no alteration in plasma glucose concentration between alcohol fed females and female controls. ${ }^{31}$ An examination of the activities of key enzymes (e.g., glucokinase, glucose-6phosphatase, glycogen phosphorylase A and others) revealed that following chronic ethanol consumption, the enzyme activities for both alcohol fed males and females were altered to the same extent and in such a manner to make glucose readily available to other tissues/organs. ${ }^{31}$ This would support the lower hepatic glycogen levels observed following chronic alcohol consumption, irrespective of sex. The authors subsequently proposed that the ability for alcohol fed females to maintain their plasma glucose levels was attributable to 
lower glucose use by peripheral tissues. ${ }^{31}$ Alternatively, in ethanol fed males, there could have been an elevated utilization of glucose by the peripheral organs/tissues contributing to the lower plasma glucose levels. ${ }^{31}$

Years later, Maly and $\mathrm{Sasse}^{32}$ reported sex differences in Wistar rats pertaining to the location of hepatic ADH. The hepatic ADH activity was preferentially higher in the perivenous zone of the liver in females versus males. They later reported in humans the same differential activities of hepatic ADH in females compared to males. ${ }^{19}$ The significance of this observation relates to the metabolic zonation of the liver reported by Jungermann and Katz. ${ }^{33}$ They proposed a physical organization where the periportal zone is primarily responsible for gluconeogenesis and the perivenous zone is primarily responsible for glycolysis based upon the location of the cells to the incoming blood. ${ }^{33}$ The liver is a unique organ that receives oxygenated blood from both the hepatic artery and portal vein, with the majority of oxygenated blood being derived from the portal vein. Given that glucose production is an energy requiring process, they proposed that the periportal hepatocytes engage in more gluconeogenesis compared to glycolysis. ${ }^{33}$ With less oxygen in the blood for the perivenous hepatocytes, these cells will primarily rely on more glycolysis compared to gluconeogenesis. However, the liver has the ability to adapt in response to various physiologic circumstances. Specifically, in situations where elevations in liver glucose production are warranted, e.g., chronic exposure to cold or acute exercise, the HGN capacity becomes selectively higher from the perivenous compared to the periportal zone, making the entire liver more homogenous as it pertains to its glucose production potential. ${ }^{34,35}$ As such, any decrement in gluconeogenic potential from the perivenous zone could result in a lower capacity for glucose production from the entire liver. The culmination of these past reports $19,31,32$ led us to suspect sex differences in glucose homeostasis following chronic alcohol consumption.

Twenty-five years after the initial study of Winston and Reitz ${ }^{31}$ in which sex differences in blood glucose homeostasis were reported, we now provide additional evidence for the interactive effects of gender and chronic alcohol consumption on blood glucose homeostasis. ${ }^{36}$ In contrast to Winston and Reitz, ${ }^{31}$ we used Wistar rats to take into account any impact attributable to the location of hepatic ADH between males versus females. We also employed a 48 -hour fast to deplete hepatic glycogen stores. For the rat, a 24 to 48 -hour fast results in a substantial decline in liver glycogen content. Further, we used dual radio-labeled glucose infusions to measure in vivo rates of whole body glucose production and apparent glucose carbon recycling (an indicator of gluconeogenesis). We subsequently injected an equivalent alcohol dose $(4 \mathrm{~g} / \mathrm{kg})$ into all chronic ethanol fed animals and corresponding controls where we followed the impact of alcohol on blood glucose levels over a 1-hour period. ${ }^{36}$ Following 8 weeks of chronic alcohol consumption, the female animals chronically fed the ethanol diet had significantly lower blood glucose levels prior to and after the injection of alcohol compared to all other groups (figure 1). Under these circumstances, hepatic glycogen stores were essentially depleted making gluconeogenesis the primary mode to resist the fall in blood glucose concentration. For all animals (both ethanol fed and controls) there was a slight decrement in blood glucose level. The fall in blood glucose concentration was attributed to a significant decline in glucose production with an earlier onset observed in ethanol fed females compared to male and female controls (figure 2). The decrease in glucose production was supported by the apparent rates of glucose carbon recycling which were significantly lower for ethanol fed females and occurred at an earlier onset compared to male and female controls. ${ }^{36}$ In contrast, ethanol fed males similarly had lower glucose production and apparent rates of glucose carbon recycling, but its occurrence was delayed compared to ethanol fed females. Further, ethanol fed male animals were able to match more closely the marked declines in glucose production with comparable reductions in glucose disappearance. ${ }^{36}$ Consistent with the observations in humans, our ethanol fed female animals demonstrated higher plasma alcohol levels. As such, we cannot rule out the possibility that the higher amount of circulating alcohol caused the dramatic fall in glucose production and/or the failure to appropriately lower peripheral glucose clearance.

Of interest, our findings are in contrast to the initial report of Winston and Reitz. ${ }^{31}$ They observed a decline in plasma glucose concentration in males chronically fed an alcohol diet, whereas we observed the decline in females. However,

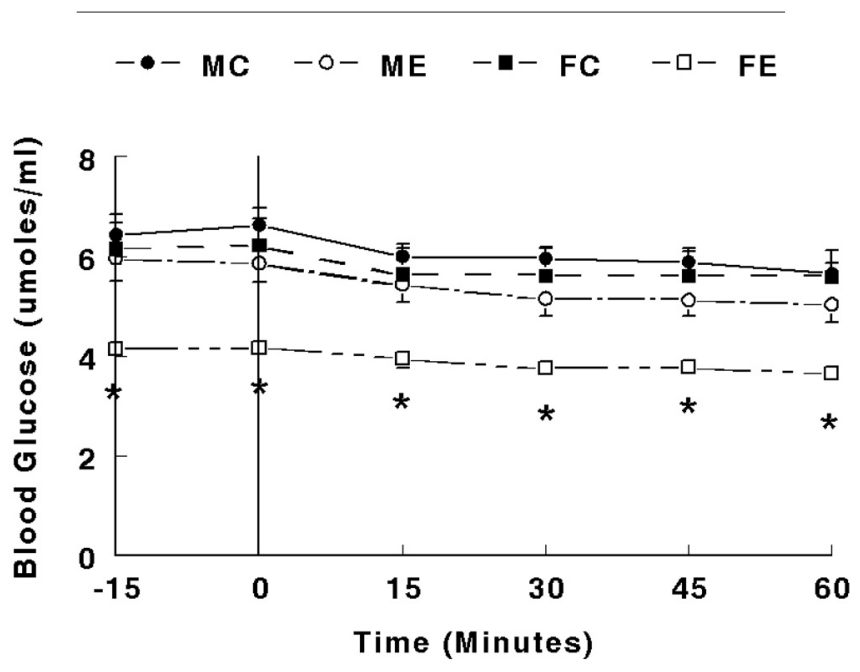

Figure 1. Effects of chronic alcohol consumption on blood glucose concentration (mean $\pm \mathrm{SE}$ ) as a function of time prior to and following the injection of ethanol for males fed the control diet (MC, $n=8)$, males fed the ethanol diet (ME, $n=8)$, females fed the control diet ( $F C, n=8)$ and females fed the ethanol diet (FE, $n=8)$. *, Significant difference between $F E$ and controls, $P<0.05$. (Reproduced from Sumida et al. Chronic alcohol consumption yields sex differences in whole-body glucose production in rats. Alcohol Alcohol 2004;39:418-426. ${ }^{36}$ ) 
we note several differences between the two studies and speculate that these differences explain the divergent results. We used fasted animals to match the nutritional deficiencies reported in alcoholics and the concomitant depletion of hepatic glycogen stores, whereas Winston and Reitz ${ }^{31}$ examined animals in the fed state. The fasted state would elevate the reliance on gluconeogenesis from the kidneys and the liver to help maintain blood glucose homeostasis. In addition, Winston and Reitz ${ }^{31}$ only examined a single blood glucose level at the time of sacrifice in the absence of an alcohol injection. In contrast, we followed the impact of alcohol on blood glucose levels over the course of 1 hour following an equivalent alcohol dose for males and females. Finally, if the location of hepatic ADH previously observed by Maly and Sasse 32 is a factor for sex differences, then it is unknown whether the sex differences in hepatic ADH exist in Long-Evans rats used by Winston and Reitz. ${ }^{31}$ Again, we used Wistar rats, consistent with Maly and Sasse, 32 to minimize differences attributable to animal strain.

Evidence for sex differences in glucose homeostasis following chronic alcohol consumption in animals has gradually been forthcoming. In the liver, the ability to mount a counter-regulatory response to hypoglycemia is primarily mediated by glucagon and $\alpha_{1}$ - and $\beta_{2}$-adrenergic receptors. Briefly, the $\alpha_{1}$-adrenergic receptors couple to two pertussis toxin G-proteins involved in phosphoinositide hydrolysis. This results in an elevation of cytosolic calcium which binds to calmodulin and catalyzes the phosphorylation of various protein kinases. $\beta_{2}$-adrenergic receptors couple to $G_{s}$, which is involved in adenylate cyclase activation and elevations in

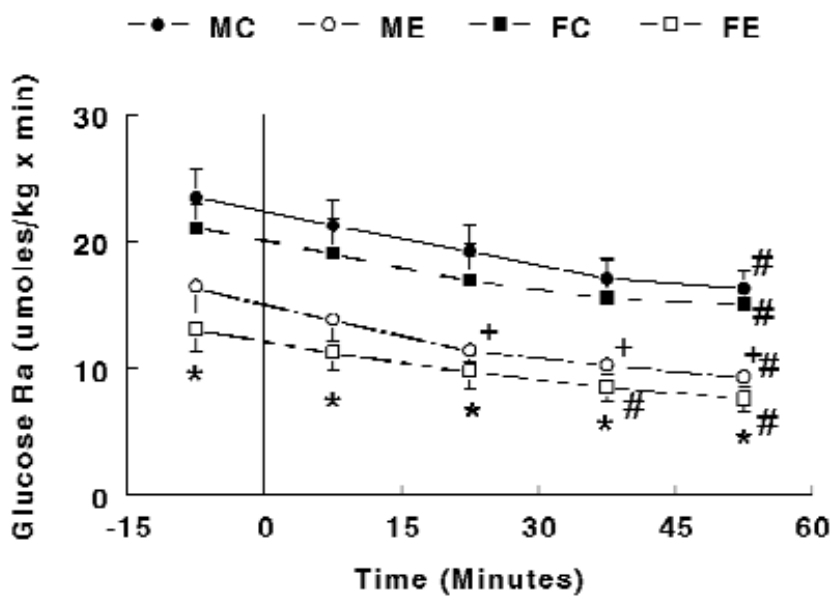

Figure 2. Effects of chronic alcohol consumption on glucose rates of appearance ( $\mathrm{Ra}$, mean $\pm \mathrm{SE}$ ) as a function of time prior to and following the injection of ethanol for males fed the control diet (MC, $n=8)$, males fed the ethanol diet (ME, $n=8)$, females fed the control diet $(F C, n=8)$ and females fed the ethanol diet (FE, $n=8)$. * Significant difference between FE and controls, $P<0.05 .+$, Significant difference between $\mathrm{ME}$ and controls, $P<0.05$. \#, Significant difference between the indicated time point and the initial level within a group, $P<0.05$. (Reproduced from Sumida et al. Chronic alcohol consumption yields sex differences in whole-body glucose production in rats. Alcohol Alcohol 2004;39:418-426. ${ }^{36}$ )
cAMP. Increased cAMP can similarly activate various protein kinases via phosphorylation. In like manner, glucagon's actions have been demonstrated to be mediated by $\mathrm{G}_{\mathrm{s}}$ using cAMP as the second messenger and the subsequent activation of various protein kinases. The culmination of various protein kinase activations, either through calcium or cAMP, is an elevation in glycogenolysis and gluconeogenesis.

Chronic alcohol consumption in rats has been observed by Gandhi and $\operatorname{Ross}^{37}$ to reduce the $\alpha_{1}$-adrenergic receptor regulation of cytosolic calcium, thereby preventing the liver from maintaining calcium levels for second messenger functions (e.g., hepatic glucose output). This would emphasize the importance of both glucagon and $\beta_{2}$ adrenergic receptors to mediate a counter-regulatory response to hypoglycemia via cAMP as the second messenger for activation of protein kinases. Lee and Hosein ${ }^{38}$ reported a lower rate of glucagon- and epinephrine-stimulated HGN from perfused livers of chronic alcohol fed rats compared to controls. They later report significant reductions in the binding of ${ }^{125} \mathrm{I}$-labeled glucagon and of $\left[{ }^{3} \mathrm{H}\right]$-prazosin to rat liver plasma membranes after chronic alcohol consumption, attributable to a decline in the density of membrane receptors. ${ }^{39,40}$ In this regard, the hepatic response to counterregulatory hormones may be diminished in alcoholics, thereby limiting their ability to combat hypoglycemia. Sex differences for humans in hepatic membrane receptors, hormone sensitivity and/or hormonal response following chronic alcohol consumption are currently unknown. However, given the alterations associated with the animal studies above and the human studies pertaining to sex differences in the counter-regulatory response to hypoglycemia (below), the potential for differential effects in males versus females who chronically consume alcohol would seem highly probable.

Evidence for Sex Differences in Counter-Regulatory Responses to Hypoglycemia

In the past, it was assumed that there were no sex differences in the counter-regulatory response to hypoglycemia. However, in two separate human studies there appears to be a sexual dimorphism in response to insulin-induced hypoglycemia.41,42 Using hypoglycemic insulin clamps, Diamond et $\mathrm{al}^{41}$ reported identical declines in plasma glucose in both men and women. Despite the equivalent decline in glucose levels, epinephrine, norepinephrine and growth hormone were significantly greater for men compared to women. ${ }^{41}$ A different study employed euglycemichypoglycemic clamps in which intravenous insulin infusions were performed, and the amount of glucose infused to maintain a specific glucose level relates to peripheral insulin sensitivity. Using this technique, Amiel et al ${ }^{42}$ demonstrated a diminished catecholamine response in women compared to men and greater peripheral insulin sensitivity in males.

Adding to the complexity is the sex difference in the distribution of adipose tissue between men and women and 
the potential impact upon glucose homeostasis. Men tend to store a greater proportion of fat in the abdominal region compared to women. ${ }^{43}$ In this regard, lipolysis appears to be greater from visceral fat deposits compared to other regions. ${ }^{44}$ As such, catecholamine-induced lipolysis results in higher circulating free fatty acid levels for men compared to women. ${ }^{45,46}$ As stated earlier, free fatty acids appear to have a permissive effect on glucose homeostasis, where an elevated level of plasma free fatty acids simultaneously provides a sparing of glucose use and stimulation of hepatic glucose production. Thus, the impact of an elevated catecholamine response in men would result in higher circulating free fatty acids, thereby providing a protection against hypoglycemia.

Studies examining the sex differences associated with acute or chronic alcohol consumption and the counter-regulatory response to alcohol-induced hypoglycemia are virtually absent. Of interest, the counter-regulatory response has been examined in men as it pertains to the acute effect of ethanol on insulin-induced hypoglycemia in which lower glucagon and cortisol secretions were noted ${ }^{47}$ However, the response in women is unknown and to our knowledge no one has investigated sex differences in the counter-regulatory response to alcohol-induced hypoglycemia. Given the sex differences in the counter-regulatory response as previously noted in the absence of alcohol, we speculate that women would demonstrate even lower counter-regulatory hormone secretions to combat alcohol-induced hypoglycemia. With that said, we note that any examination of sex differences in the counter-regulatory response to hypoglycemia would need to consider the amount of alcohol administered and the potential for differences in blood alcohol levels. From the preliminary information we have to date, we would similarly anticipate that the counter-regulatory response in alcoholic women could be even lower compared to alcoholic men to combat alcohol-induced hypoglycemia, but this remains to be determined. Further, any sex differences in the counterregulatory response to hypoglycemia in alcohol drinkers would need to consider the amount of ethanol chronically consumed, i.e., light/moderate versus heavy.

Our in vivo observation of a decline in whole body glucose production in ethanol fed females is supported, collectively, by these studies that demonstrate a lower release of counterregulatory hormones in response to hypoglycemia in females, the hepatic decline in membrane receptors for the counterregulatory hormones reported in alcoholic animals, as well as the sex differences in lipolysis associated with regional adipose deposits in humans. This would suggest that alcoholic females would be more vulnerable to alcohol-induced hypoglycemia than males. Unfortunately, the site for the diminution in whole body glucose production was beyond the scope of our prior in vivo study, but suggests either the kidneys and/or the liver.

\section{Evidence for Sex Differences in HGN Capacity}

Consistent with prior studies, our focus for the decline in

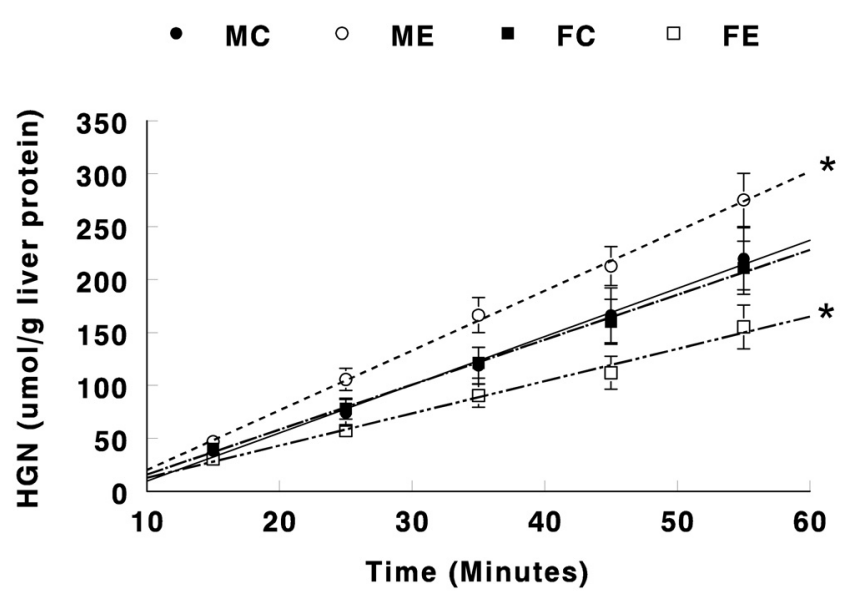

Figure 3. Effect of chronic alcohol consumption on hepatic gluconeogenesis (HGN) from lactate versus time from males fed the control diet (MC, $n=6$ ), males fed the ethanol diet (ME, $\mathrm{n}=6)$, females fed the control diet $(\mathrm{FC}, \mathrm{n}=6)$ and females fed the ethanol diet $(F E, n=6)$. *Significant difference in slope between FE vs. controls and ME vs. controls, $P<0.05$. (Reprinted with permission from Sumida et al. Opposing effects of chronic alcohol consumption on hepatic gluconeogenesis for female versus male rats. Alcohol Clin Exp Res 2005;29:1899-1905. ${ }^{48}$ Copyright 2005 Blackwell Publishing Ltd. All rights reserved.)

whole body glucose production was the liver. As such, our recent findings have repeatedly demonstrated that chronic alcohol consumption by female rats differentially impacts HGN capacity compared to chronic alcohol consumption by male rats. The sex differences were demonstrated using a variety of techniques, including in situ liver perfusions, isolated hepatocytes, and measuring the enzyme activities of $\mathrm{ADH}$ and lactate dehydrogenase.

Using the in situ isolated liver perfusion technique from 24-hour fasted Wistar rats, rates of glucose production from lactate were measured from the livers of ethanol fed females, ethanol fed males and corresponding female and male controls..$^{48}$ There were no significant differences in rates of HGN between male and female controls. In contrast, after 8 weeks of the chronic ethanol diet, ethanol fed females had significantly lower gluconeogenic rates (figure 3). While ethanol fed males had significantly higher HGN, we also report a higher triglyceride content in the ethanol fed male livers and attribute the increased HGN to the elevated triglyceride content which has been shown to stimulate glucose production. ${ }^{15}$ Consistent with the decline in HGN capacity, lactate uptake was significantly lower for ethanol fed females. ${ }^{48}$ We also perfused the liver with ${ }^{14} \mathrm{C}$-lactate to help confirm any alteration in HGN. ${ }^{48}$ We observed lower incorporation of ${ }^{14} \mathrm{C}$-lactate into ${ }^{14} \mathrm{C}$-glucose, as well as lower ${ }^{14} \mathrm{C}$-lactate uptake rates in ethanol fed females, supporting the reduced HGN capacity in ethanol fed females. ${ }^{48}$ These findings provide the first direct evidence of a decline in HGN capacity after chronic alcohol consumption in female animals in the absence of circulating ethanol. 
Since the liver perfusion studies discussed above did not examine the impact of alcohol in the perfusion medium, we subsequently investigated the impact of ethanol on HGN after 8 weeks of chronic alcohol consumption in male versus female Wistar rats. ${ }^{49}$ After fasting the animals for 24 hours to again deplete hepatic glycogen, we employed the isolated hepatocyte technique. ${ }^{49}$ Aliquots of the liver cell suspensions were placed in Krebs-Henseleit buffer and incubated for 30 minutes with lactate, $\left[\mathrm{U}-{ }^{14} \mathrm{C}\right]$-lactate and 9 different concentrations of ethanol. There was no significant difference in HGN (lactate only and no ethanol) between males and females fed the control diet (figure 4). Similarly, the HGN (lactate only and no ethanol) in males fed the ethanol diet was not significantly different compared to controls. ${ }^{49}$ In contrast, the females chronically fed the ethanol diet had significantly lower HGN (figure 5) compared to both ethanol fed males and controls. ${ }^{49}$ With alcohol in the incubation medium, the HGN significantly declined in all groups (figure 4). While alcohol suppressed HGN to a larger extent in ethanol fed males compared to controls, the inhibition was even greater in ethanol fed females (figure 5). The more pronounced effect of chronic alcohol consumption on HGN in the absence and presence of ethanol in female hepatocytes was supported by the concomitant decreases in lactate uptake (figure 5), ${ }^{14} \mathrm{C}$ lactate incorporation into ${ }^{14} \mathrm{C}$-glucose, and ${ }^{14} \mathrm{C}$-lactate uptake. ${ }^{49}$ These observations suggest that chronic alcohol consumption elicits a greater reduction on HGN in the presence of ethanol in the hepatocytes of females compared to males.

While others might have similarly investigated the potential for chronic ethanol consumption to elicit an effect upon

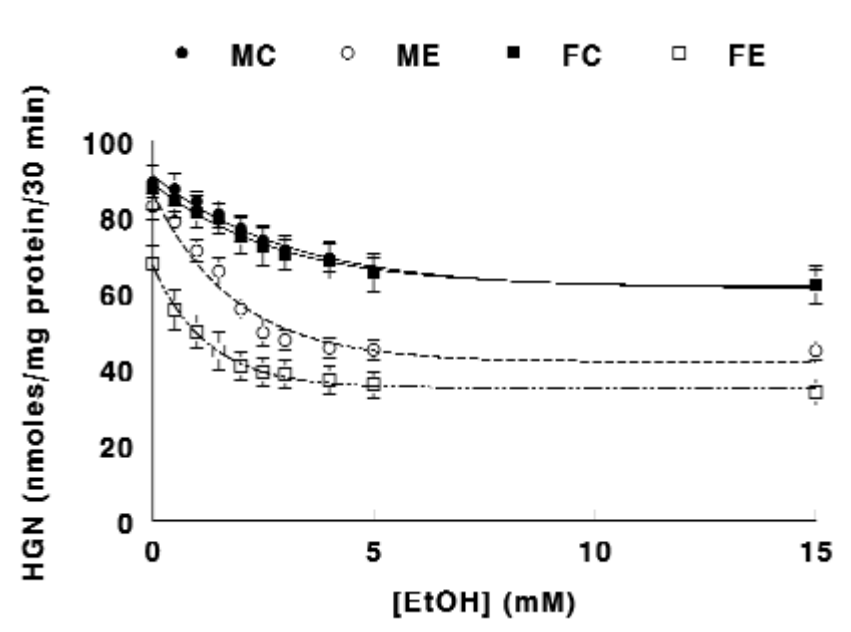

Figure 4. Effect of various concentrations of alcohol on hepatic gluconeogenesis (HGN) in hepatocytes of males fed the control diet $(M C, n=8)$, males fed the ethanol diet (ME, $\mathrm{n}=8$ ), females fed the control diet (FC, $\mathrm{n}=8$ ) and females fed the ethanol diet $(F E, n=8)$. The correlation coefficients were $>0.9$ for all groups. (Reproduced with permission from Sumida et al. Alcohol-induced suppression of gluconeogenesis is greater in ethanol fed female rat hepatocytes than males. Alcohol 2007;41:67-75. ${ }^{49}$ Copyright 2007 Elsevier. All rights reserved.)

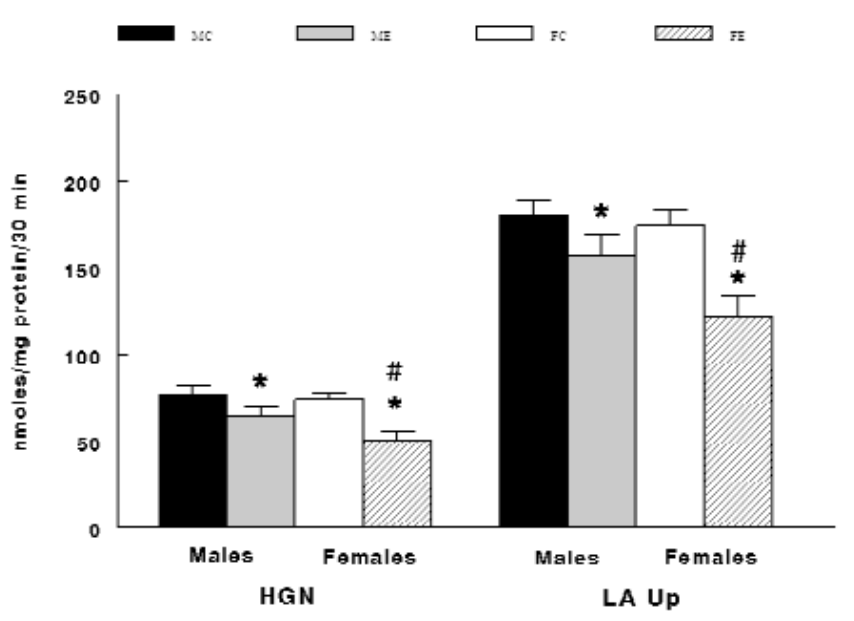

Figure 5. Interaction between sex and diet on hepatic gluconeogenesis (HGN) and lactate uptake (LA Up) in hepatocytes of males fed the control diet (MC, $n=8)$, males fed the ethanol diet (ME, $n=8)$, females fed the control diet ( $F C$, $\mathrm{n}=8$ ) and females fed the ethanol diet $(F E, n=8)$. *, Significant difference between $M C$ vs. ME and between FC vs. FE, $P<0.05$. \#, Significant difference between ME vs. FE, $P<0.05$. (Reproduced with permission from Sumida et al. Alcoholinduced suppression of gluconeogenesis is greater in ethanol fed female rat hepatocytes than males. Alcohol 2007;41:6775. ${ }^{49}$ Copyright 2007 Elsevier. All rights reserved.)

glucose production capacity in the liver, the impact may not have been observed due to the tendency to use men, or male animals, in the experimental studies. Further, to the extent that $\mathrm{ADH}$ is a factor for sex differences, another potential reason for the lack of reports could be the use of an animal model that is not comparable to humans pertaining to the location of hepatic ADH. Thus, in all the studies we performed, we used Wistar rats. In addition and as mentioned throughout this review, a multitude of factors between men and women could also influence the impact of chronic alcohol consumption on glucose homeostasis, making sex differences difficult to assess. As another example, it is possible that the length of chronic alcohol consumption and/or the fat content in the alcohol diet might be additional factors dictating the impact within the liver. In support, we observed an elevated triglyceride content in the livers from ethanol fed males compared to ethanol fed females, whereas an elevated accumulation of fat has previously been reported in chronic ethanol fed females compared to males. ${ }^{21}$ We have no explanation for the discrepancy, but we note two distinct differences between the studies that could support our contention pertaining to the length of chronic alcohol consumption and the fat content within the diet. Specifically, we examined animals chronically fed an ethanol diet for 8 weeks, whereas others ${ }^{21}$ examined the impact of alcohol consumption after 4 weeks. Thus, it is possible that more time might yield an adaptation within the liver of ethanol fed females bringing the triglyceride content down, whereas the males elicit a delayed response to chronic alcohol consumption for the hepatic deposition of triglycerides. Also, in the liver perfusion study, ${ }^{48}$ we failed to use a low-fat ethanol diet and subsequently observed a greater triglyceride 
content in male livers. However, in our isolated hepatocyte report, ${ }^{49}$ we employed the use of a low fat ethanol diet. As such, we note that only in the liver perfusion study did we observe greater HGN in ethanol fed males, supporting the enhanced effects of fatty acids on glucose production capacity. In contrast, we failed to observe an elevated HGN when male animals ingested the low fat ethanol diet. However, irrespective of the fat content within the diet, ethanol fed females demonstrated lower HGN in both isolated hepatocytes ${ }^{49}$ and intact liver, ${ }^{48}$ which emphasizes the deleterious effect of chronic alcohol consumption within the livers of female animals.

The mechanism for the significant decline in HGN capacity in females compared to males after chronic alcohol consumption, in both the absence and presence of ethanol, was beyond the scope of our previous studies. However, we did examine the total hepatic enzyme activities of both ADH and lactate dehydrogenase (LDH). ${ }^{48}$ We reported higher hepatic ADH activities in females compared to males irrespective of ethanol consumption (figure 6). In addition, chronic alcohol consumption lowered ADH activity in the liver. ${ }^{48}$ While this observation is consistent with previous reports, ${ }^{50,51}$ it deserves some consideration given the higher plasma alcohol levels we also observed from ethanol fed female animals in our in vivo study. In this regard, given the higher ADH activity for ethanol fed females, ostensibly the plasma alcohol levels should be lower. Given the absence of gastric ADH reported in humans for alcoholic women, our results in female animals would support a decline in the first pass metabolism of alcohol resulting in a higher blood alcohol level. It should also be noted that the liver can metabolize alcohol with use of the enzyme cytochrome P450 (Cyp) 2E1. In this regard, animal studies suggest that the hepatic enzyme activity is greater in males than females. ${ }^{52,53}$ After a period of chronic alcohol consumption in rats, the activity of the Cyp2E1 enzyme increases, but continues to remain lower for females compared to males. ${ }^{52,54}$ This would further support our observation of higher plasma alcohol levels for ethanol fed females. Of interest, there appears to be no sex difference for hepatic Cyp2E1 in humans. ${ }^{55}$ While we did not measure Cyp2E1 activity from livers of the animals in our study, we did measure hepatic LDH, an enzyme indirectly involved with the gluconeogenic pathway.

LDH was significantly lower in ethanol fed females compared to corresponding controls, whereas no significant decline was observed for ethanol fed males. In this regard, we speculate that the decline in $\mathrm{ADH}$ is greatest in the hepatic region where it is found. Thus, it is possible that the corresponding decline in LDH activity in ethanol fed females might similarly take place in the same hepatic region in an attempt to normalize the redox state. If this occurred, then the decrease in LDH in the perivenous region could result in a lower ability to convert lactate to pyruvate, culminating in the decrement in gluconeogenic capacity in the perivenous region of females. Although this may seem insignificant, we again

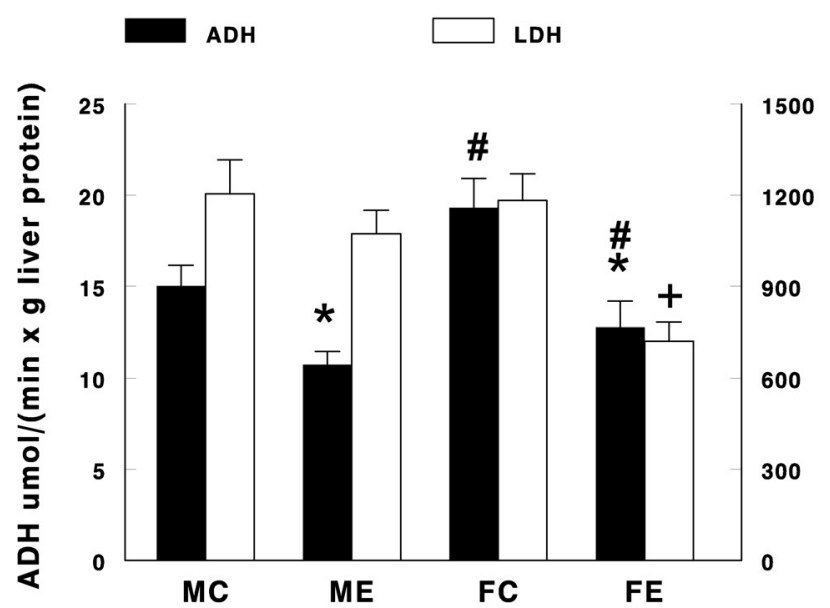

Figure 6. Total hepatic alcohol dehydrogenase activity (ADH) and total hepatic lactate dehydrogenase (LDH) activity from males fed the control diet $(\mathrm{MC}, \mathrm{n}=8)$, males fed the ethanol diet ( $M E, n=8)$, females fed the control diet $(F C, n=8)$ and females fed the ethanol diet (FE, $n=8)$. For ADH: *, significant difference between ethanol fed animals vs. corresponding controls, $P<0.05$. \#, Significant difference between females vs. males for control and ethanol fed animals, $P<0.05$. For LDH: +, significant difference between FE vs. all other groups, $P<0.05$. (Reprinted with permission from Sumida et al. Opposing effects of chronic alcohol consumption on hepatic gluconeogenesis for female versus male rats. Alcohol Clin Exp Res 2005;29:1899-1905..8 Copyright 2005 Blackwell Publishing Ltd. All rights reserved.)

note that the perivenous region of the liver would normally increase its glucose production capacity, resulting in a more homogeneous response to help prevent hypoglycemia. Alternatively, it is possible that chronic alcohol consumption (where blood alcohol levels tend to be higher in females despite an equivalent ethanol load) might differentially alter the hepatic plasma membrane which might affect monocarboxylate transporter number and/or impact the activity of other enzymes involved with the gluconeogenic pathway. Finally, we cannot rule out the possibility that sex differences after chronic alcohol consumption, either in the absence or presence of ethanol, might similarly exist in the kidneys and/or periphery (e.g., skeletal muscle glucose uptake), resulting in alterations in glucose homeostasis. Another unknown factor that we previously noted and that remains to be determined is the impact of chronic alcohol consumption and gender differences in the counter-regulatory response to ethanol-induced hypoglycemia.

\section{Glucose Homeostasis - Implications for Alcoholics}

Evidence for sex differences in glucose homeostasis after chronic alcohol consumption in animals has been gradually forthcoming for the past 25 years. We now provide direct evidence for a specific impact upon ethanol fed female rats compared to males. Following a fast, females chronically fed an alcohol diet demonstrated a decline in whole body glucose production prior to and after an injection of ethanol compared to controls. This suggests a decrement in the gluconeogenic 
capacity of the kidneys and/or the liver which are the organs responsible for glucose homeostasis under fasting conditions. In follow-up studies, we determined that the source for at least some of the decline in whole body glucose production emanates from the liver. Specifically, after chronic alcohol consumption, 24 hour fasted female rats demonstrate a lower gluconeogenic capacity from lactate in the absence of ethanol that is exacerbated in the presence of alcohol. While the mechanism for the reduction in gluconeogenic capacity within the liver remains to be elucidated, sex differences in the location of $\mathrm{ADH}$ and the diminution in LDH activity in females fed an alcohol diet might be one factor among many contributing to the decline.

A thorough examination of our prior studies $36,48,49$ will reveal several limitations that we acknowledge. Further, our studies primarily focused on the liver. ${ }^{48,49}$ Whether there are similar sex differences in the gluconeogenic capacity of the kidneys, alterations in skeletal-muscle-glucose uptake and/or sexual dimorphism in the counter-regulatory response to alcoholinduced hypoglycemia remain to be investigated. In addition, other relevant factors for ethanol-induced hypoglycemia that remain to be determined in humans include ethnicity, menstrual cycle and/or status (i.e., menopause) and age. Further, there are sex differences reported in animals for the detoxification of alcohol (e.g., Cyp2E1) that are not observed in humans, as well as sex differences in humans (e.g., regional adiposity) that are not clearly delineated in animals. As such, we recognize that multiple factors are involved in glucose homeostasis. However, to the extent that our results from the liver in rats can be extrapolated to humans, the vulnerability for ethanol-induced hypoglycemia would conceivably be higher for alcoholic women compared to men. While there is a lack of human reports to support this contention, we hope this review will help clinicians to be aware of the potential for sex differences in glucose homeostasis in alcoholics.

\section{References}

1. Auer RN. Progress review: hypoglycemic brain damage. Stroke 1986;17:699-708.

2. Freinkel N, Singer DL, Arky RA, Bleicher SJ, Anderson JB, Silbert CK. Alcohol hypoglycemia. I. Carbohydrate metabolism of patients with clinical alcohol hypoglycemia and the experimental reproduction of the syndrome with pure ethanol. J Clin Invest 1963;42:1112-1133.

3. Krebs HA. The effects of ethanol on the metabolic activities of the liver. Adv Enzyme Regul 1968;6:467-480.

4. Krebs HA, Freedland RA, Hems R, Stubbs M. Inhibition of hepatic gluconeogenesis by ethanol. Biochem J 1969;112:117-124.

5. Searle GL, Shames D, Cavalieri RR, Bagdade JD, Porte D Jr. Evaluation of ethanol hypoglycemia in man: turnover studies with C-6 14C glucose. Metabolism 1974;23:1023-1035.

6. Wolfe BM, Havel JR, Marliss EB, Kane JP, Seymour J, Ahuja SP. Effects of a 3-day fast and of ethanol on splanchnic metabolism of FFA, amino acids, and carbohydrates in healthy young men. J Clin Invest 1976;57:329-340.
7. Wilson NM, Brown PM, Juul SM, Prestwich SA, Sonksen PH. Glucose turnover and metabolic and hormonal changes in ethanol-induced hypoglycaemia. Br Med J (Clin Res Ed) 1981;282:849-853.

8. Oliveira de Souza ML, Masur J. Blood glucose and body temperature alterations induced by ethanol in rats submitted to different levels of food deprivation. Pharmacol Biochem Behav 1981;15:551-554.

9. Souza ML, Masur J. Does hypothermia play a relevant role in the glycemic alterations induced by ethanol? Pharmacol Biochem Behav 1982;16:903-908.

10. Souza ML, Masur J. Ethanol induces hyper and hypoglycemia in both fasted and nonfasted rats dependent on the ambient temperature. Pharmacol Biochem Behav 1984;20:649-652.

11. Salaspuro M. Nutrient intake and nutritional status in alcoholics. Alcohol Alcohol 1993;28:85-88.

12. Addolorato G, Capristo E, Greco AV, Stefanini GF, Gasbarrini G. Influence of chronic alcohol abuse on body weight and energy metabolism: is excess ethanol consumption a risk factor for obesity or malnutrition? J Intern Med 1998;244:387-395.

13. Ylikahri RH, Huttunen MO, Harkonen M. Hormonal changes during alcohol intoxication and withdrawal. Pharmacol Biochem Behav 1980;13:131-137.

14. Avogaro A, Valerio A, Miola M, Crepaldi C, Pavan P, Tiengo A, del Prato S. Ethanol impairs insulin-mediated glucose uptake by an indirect mechanism. J Clin Endocrinol Metab 1996;81:2285-2290.

15. Berry MN, Gregory RB, Grivell AR, Henly DC, Phillips JW, Wallace PG, Welch GR. Evidence that stimulation of gluconeogenesis by fatty acid is mediated through thermodynamic mechanisms. FEBS Lett 1988;231:19-24.

16. Freiberg MS, Cabral HJ, Heeren TC, Vasan RS, Curtis Ellison R; Third National Health and Nutrition Examination Survey. Alcohol consumption and the prevalence of the Metabolic Syndrome in the US: a cross-sectional analysis of data from the Third National Health and Nutrition Examination Survey. Diabetes Care 2004;27:2954-2959.

17. Djousse L, Arnett DK, Eckfeldt JH, Province MA, Singer MR, Ellison RC. Alcohol consumption and metabolic syndrome: does the type of beverage matter? Obes Res 2004;12:13751385.

18. Fan AZ, Russell M, Dorn J, Freudenheim JL, Nochajski T, Hovey K, Trevisan M. Lifetime alcohol drinking pattern is related to the prevalence of metabolic syndrome. The Western New York Health Study (WNYHS). Eur J Epidemiol 2006;21:129-138.

19. Maly IP, Sasse D. Intraacinar profiles of alcohol dehydrogenase and aldehyde dehydrogenase activities in human liver. Gastroenterology 1991;101:1716-1723.

20. Baraona E, Abittan CS, Dohmen K, Moretti M, Pozzato G, Chayes ZW, Schaefer C, Lieber CS. Gender differences in pharmacokinetics of alcohol. Alcohol Clin Exp Res 2001;25:502-507.

21. Shevchuk O, Baraona E, Ma XL, Pignon JP, Lieber CS. Gender differences in the response of hepatic fatty acids and cytosolic fatty acid-binding capacity to alcohol consumption in rats. Proc Soc Exp Biol Med 1991;198:584-590.

22. Becker U, Deis A, Sorensen TI, Gronbaek M, Borch-Johnsen K, Muller CF, Schnohr P, Jensen G. Prediction of risk of liver disease by alcohol intake, sex, and age: a prospective population study. Hepatology 1996;23:1025-1029.

23. Teschke R, Wiese B. Sex-dependency of hepatic alcohol metabolizing enzymes. J Endocrinol Invest 1982;5:243-250.

24. Frezza M, di Padova C, Pozzato G, Terpin M, Baraona E, Lieber CS. High blood alcohol levels in women. The role of decreased gastric alcohol dehydrogenase activity and firstpass metabolism. N Engl J Med 1990;322:95-99. 
25. Lieber CS. Ethnic and gender differences in ethanol metabolism. Alcohol Clin Exp Res 2000;24:417-418.

26. Van Thiel DH, Tarter RE, Rosenblum E, Gavaler JS. Ethanol, its metabolism and gonadal effects: does sex make a difference? Adv Alcohol Subst Abuse 1988;7:131-169.

27. Seitz HK, Egerer G, Simanowski UA, Waldherr R, Eckey R, Agarwal DP, Goedde HW, von Wartburg JP. Human gastric alcohol dehydrogenase activity: effect of age, sex, and alcoholism. Gut 1993;34:1433-1437.

28. Owen OE, Reichle FA, Mozzoli MA, Kreulen T, Patel MS, Elfenbein IB, Golsorkhi M, Chang KH, Rao NS, Sue HS, Boden G. Hepatic, gut, and renal substrate flux rates in patients with hepatic cirrhosis. J Clin Invest 1981;68:240252.

29. Perdigoto R, Furtado AL, Porto A, Rodrigues TB, Geraldes CF, Jones JG. Sources of glucose production in cirrhosis by $2 \mathrm{H} 2 \mathrm{O}$ ingestion and $2 \mathrm{H}$ NMR analysis of plasma glucose. Biochim Biophys Acta 2003;1637:156-163.

30. Brancati FL, Kao WH, Folsom AR, Watson RL, Szklo M. Incident type 2 diabetes mellitus in African American and white adults: the Atherosclerosis Risk in Communities Study. JAMA 2000;283:2253-2259.

31. Winston GW, Reitz RC. Effects of chronic ethanol ingestion on glucose homeostasis in males and females. Life Sci 1980;26:201-209.

32. Maly IP, Sasse D. Microquantitative determination of the distribution patterns of alcohol dehydrogenase activity in the liver of rat, guinea-pig and horse. Histochemistry 1985;83:431-436.

33. Jungermann K, Katz N. Functional specialization of different hepatocyte populations. Physiol Rev 1989;69:708-764.

34. Shiota M, Fujimoto Y, Inagami M, Hiramatsu M, Moriyama M, Kimura K, Ohta M, Sugano T. Adaptive changes in zonation for gluconeogenic capacity in liver lobules of cold-exposed rats. Am J Physiol 1993;265:E559-E564.

35. Desy F, Burelle Y, Belanger P, Gascon-Barre M, Lavoie JM. Effects of acute exercise on the gluconeogenic capacity of periportal and perivenous hepatocytes. J Appl Physiol 2001;91:1099-1104.

36. Sumida KD, Qureshi T, Catanzaro MJ, Arimoto SM, Hill JM. Chronic alcohol consumption yields sex differences in whole-body glucose production in rats. Alcohol Alcohol 2004;39:418-426.

37. Gandhi VC, Ross DH. Effects of alcohol on alpha-adrenergic receptor regulation of calcium ATPase in liver plasma membranes. Alcohol 1987;4:25-30.

38. Lee H, Hosein EA. Effect of chronic alcohol administration on the hormonal sensitivity of isolated perfused rat liver. Life Sci 1981;29:135-141.

39. Lee H, Hosein EA. Chronic alcohol feeding and its withdrawal on the structure and function of the rat liver plasma membrane: a study with 125I-labelled glucagon binding as a metabolic probe. Can J Physiol Pharmacol 1982;60:11711176.

40. Lee H, Hosein EA, Rovinski B. Effect of chronic alcohol feeding and withdrawal on rat liver plasma membrane structure and function: a study of binding of $[3 \mathrm{H}]$ prazosin to the membrane bound alpha 1-adrenergic receptor. Biochem Pharmacol 1983;32:1321-1323.

41. Diamond MP, Jones T, Caprio S, Hallarman L, Diamond MC, Addabbo M, Tamborlane WV, Sherwin RS. Gender influences counterregulatory hormone responses to hypoglycemia. Metabolism 1993;42:1568-1572.

42. Amiel SA, Maran A, Powrie JK, Umpleby AM, Macdonald IA. Gender differences in counterregulation to hypoglycaemia. Diabetologia 1993;36:460-464.
43. Votruba SB, Jensen MD. Sex differences in abdominal, gluteal, and thigh LPL activity. Am J Physiol Endocrinol Metab 2007;292:E1823-E1828. Epub 2007 Feb 20.

44. Jensen MD. Lipolysis: contribution from regional fat. Annu Rev Nutr 1997;17:127-139.

45. Shadid S, Kanaley JA, Sheehan MT, Jensen MD. Basal and insulin regulated free fatty acid and glucose metabolism in humans. Am J Physiol Endocrinol Metab 2007;292:E1770E1774. Epub 2007 Feb 13.

46. Lonnqvist F, Thome A, Nilsell K, Hoffstedt J, Arner P. A pathogenic role of visceral fat beta 3-adrenoceptors in obesity. J Clin Invest 1995;95:1109-1116.

47. Kolaczynski JW, Ylikahri R, Harkonen M, Koivisto VA. The acute effect of ethanol on counterregulatory response and recovery from insulin-induced hypoglycemia. J Clin Endocrinol Metab 1988;67:384-388.

48. Sumida KD, Cogger AA, Arimoto SM, Matveyenko AV. Opposing effects of chronic alcohol consumption on hepatic gluconeogenesis for female versus male rats. Alcohol Clin Exp Res 2005;29:1899-1905.

49. Sumida KD, Cogger AA, Matveyenko AV. Alcohol-induced suppression of gluconeogenesis is greater in ethanol fed female rat hepatocytes than males. Alcohol 2007;41:67-75. Epub 2006 Apr 26.

50. Cascales C, Cascales M, Santos-Ruiz A. Effect of chronic ethanol or acetaldehyde on hepatic alcohol and aldehyde dehydrogenases, aminotransferases and glutamate dehydrogenase. Rev Esp Fisiol 1985;41:19-27.

51. Vaananen H, Salaspuro M, Lindros K. The effect of chronic ethanol ingestion on ethanol metabolizing enzymes in isolated periportal and perivenous rat hepatocytes. Hepatology 1984;4:862-866.

52. Van de Wiel JA, Fijneman PH, Teeuw KB, Van Ommen B, Noordhoek J, Bos RP. Influence of long-term ethanol treatment on rat liver biotransformation enzymes. Alcohol 1993;10:397-402.

53. Nebbia C, Dacasto M, Ceppa L, Bosia S, Burdino E, Witkamp RF, Ugazio G. Gender differences in ethanol oxidation and cytochrome P4502E1 content and functions in hepatic microsomes from alcohol-preferring and non-preferring rats. Xenobiotica 1996;26:1121-1129.

54. Ma X, Baraona E, Lieber CS. Alcohol consumption enhances fatty acid omega-oxidation, with a greater increase in male than in female rats. Hepatology 1993;18:1247-1253.

55. Parkinson A, Mudra DR, Johnson C, Dwyer A, Carroll KM. The effects of gender, age, ethnicity, and liver cirrhosis on cytochrome P450 enzyme activity in human liver microsomes and inducibility in cultured human hepatocytes. Toxicol Appl Pharmacol 2004;199:193-209.

\section{Author Affiliations}

Ken D. Sumida, PhD

Department of Biological Sciences

Chapman University

Orange, CA 92866

Janeen M. Hill, PhD

Department of Biological Sciences

Chapman University

Orange, CA 92866

Aleksey V. Matveyenko, PhD

Department of Biological Sciences

Chapman University

Orange, CA 92866 